\title{
On Symmetric Extended Generalized Logistic Distribution
}

\author{
Akintayo K. Olapade \\ Obafemi Awolowo University
}

\begin{abstract}
In this paper, we consider a form of the generalized logistic distribution named symmetric extended generalized logistic distribution or extended type III generalized logistic distribution. The distribution is derived by compounding a two-parameter generalized Gumbel distribution with a two-parameter generalized gamma distribution. The cumulative distribution and some properties of this distribution like moments and related statistics are established. Some theorems that characterize the distribution are stated and proved. Estimation of the parameters and an application of the distribution are also presented.
\end{abstract}

Keywords. Symmetric extended generalized logistic distribution; Moment; Gamma distribution; Gumbel distribution; Homogeneous differential equation.

\section{Introduction}

The probability density function of a random variable that has logistic distribution is

$$
f_{X}(x)=\frac{\exp \{-x\}}{(1+\exp \{-x\})^{2}}, \quad-\infty<x<\infty
$$

and the corresponding cumulative distribution function is given by

$$
F_{X}(x)=(1+\exp \{-x\})^{-1}, \quad-\infty<x<\infty .
$$


The importance of the logistic distribution has already been felt in many areas of human endeavour. Verhulst (1845) used it in economic and demographic studies. Berkson $(1944,1951,1953)$ used the distribution extensively in analyzing bio-assay and quantal response data. George and Ojo (1980), Ojo (1989), Ojo (1997), Ojo (2002), Ojo and Olapade (2004), Olapade (2002), Olapade (2004), Olapade (2005), Olapade and Ojo (2002) are few of many publications on logistic distribution.

The simplicity of logistic distribution and its importance as a growth curve have made it one of the many important statistical distributions. The shape of logistic distribution that is similar to that of normal distribution makes it simpler and also profitable on suitable occasions to replace normal distribution with logistic distribution with negligible errors in respective theories.

Balakrishnan and Leung (1988) show the probability density function of a random variable $X$ that has type III generalized logistic distribution. It is given by

$$
f_{X}(x ; b)=\frac{1}{B(b, b)} \cdot \frac{\exp \{-b x\}}{(1+\exp \{-x\})^{2 b}}, \quad-\infty<x<\infty, \quad b>0,
$$

where $B(b, b)=[\Gamma(b)]^{2} / \Gamma(2 b)$. The corresponding cumulative distribution function is

$$
F_{X}(x ; b)=\frac{1}{B(b, b)} \int_{-\infty}^{x} \frac{\exp \{-b t\}}{(1+\exp \{-t\})^{2 b}} d t .
$$

After the transformation of $z=1 /(1+\exp \{-t\})$, we have

$$
F_{X}(x ; b)=\frac{1}{B(b, b)} \int_{0}^{\frac{1}{1+\exp \{-x\}}} z^{b-1}(1-z)^{b-1} d z=I_{\frac{1}{1+\exp \{-x\}}}(b, b)
$$

in which the integral is known as incomplete beta function. Since the function $F_{X}(x ; b)$ cannot be expressed in terms of a simple formula involving $b$ and $x$, it must be tabulated and a separate table is needed for every value of parameter $b$, a complete book of tables is required to give $F_{X}(x ; b)$ for all $b$ that might be met in practice. One of such books is tables of the Incomplete Beta function, edited by Pearson (1934). In these tables, we take $b=p=q$. The integral in the equation (2) could easily be evaluated especially by using computer programs like Maple V, Mathematical etc when $b$ is specified for any value of the real number $x$.

In this paper, we will derive a generalized logistic distribution function that generalizes the type III generalized logistic distribution of Balakrishnan and Leung (1988) and is a particular case of the general case considered in $\mathrm{Wu}$, Hung and Lee (2000) and Ojo and Olapade (2004). The new density function 
is called symmetric extended generalized logistic distribution or extended type III generalized logistic distribution.

\section{$2 \quad$ Symmetric Extended Generalized Logistic Distribution}

Let $X$ be a continuously distributed random variable with two-parameter Gumbel probability density function

$$
f_{X}(x ; \alpha, p)=\frac{\alpha^{p}}{\Gamma(p)} \exp \{-p x\} \exp \left(-\alpha e^{-x}\right), \quad-\infty<x<\infty, \quad p>0, \quad \alpha>0 .
$$

Assuming that $\alpha$ has a gamma distribution with probability density function

$$
h(\alpha ; \lambda, p)=\frac{\lambda^{p}}{\Gamma(p)} \alpha^{p-1} \exp (-\lambda \alpha), \quad p>0, \quad \lambda>0 .
$$

Then we obtain the probability density of the compound distribution based on the equations (3) and (4) as

$$
\begin{aligned}
f_{X}(x ; \lambda, p) & =\int_{0}^{\infty} f_{X}(x ; \alpha, p) h(\alpha ; \lambda, p) d \alpha \\
& =\frac{\lambda^{p}}{B(p, p)} \cdot \frac{\exp \{-p x\}}{(\lambda+\exp \{-x\})^{2 p}}, \quad-\infty<x<\infty, \quad \lambda>0, \quad p>0 .
\end{aligned}
$$

We refer to this density function in the equation (5) as the symmetric extended generalized logistic distribution or the extended type III generalized logistic distribution. The case $\lambda=1$ corresponds to the type III generalized logistic distribution in Balakrishnan and Leung (1988), while the case $p=\lambda=1$ gives the ordinary logistic density function given in the equation (1).

\subsection{The Cumulative Distribution Function of the Ex- tended Type III Generalized Logistic Distribution}

The cumulative distribution function of the extended type III generalized logistic distribution is obtained as

$$
F_{X}(x ; \lambda, p)=\frac{\lambda^{p}}{B(p, p)} \int_{-\infty}^{x} \frac{\exp \{-p t\}}{(\lambda+\exp \{-t\})^{2 p}} d t
$$


The above integral does not have a closed form, hence it is obtained numerically using Maple $\mathrm{V}$ computer programme. As the values of $F_{X}(x ; \lambda, p)$ depend on the values of $p, \lambda$ and $x$, some values of $F_{X}(x ; \lambda, p)$ are tabulated in Table 1.

Table 1. The cumulative distribution of the extended type III generalized logistic distribution.

\begin{tabular}{ccccccccccc}
\multicolumn{10}{c}{$p=1, \lambda=2$} \\
\hline \hline $\mathbf{x}$ & $\mathbf{. 0 0}$ &. $\mathbf{1 0}$ &. $\mathbf{2 0}$ & $\mathbf{. 3 0}$ &. $\mathbf{4 0}$ & $\mathbf{. 5 0}$ & $\mathbf{. 6 0}$ & $\mathbf{. 7 0}$ & $\mathbf{. 8 0}$ & $\mathbf{. 9 0}$ \\
\hline $\mathbf{. 0}$ & .6667 & .6885 & .7095 & .7297 & .7490 & .7673 & .7847 & .8011 & .8166 & .8311 \\
$\mathbf{1 . 0}$ & .8446 & .8573 & .8691 & .8801 & .8902 & .8996 & .9083 & .9163 & .9237 & .9304 \\
$\mathbf{2 . 0}$ & .9366 & .9423 & .9475 & .9523 & .9566 & .9606 & .9642 & .9675 & .9705 & .9732 \\
$\mathbf{3 . 0}$ & .9757 & .9780 & .9800 & .981 .9 & .9836 & .9851 & .9865 & .9878 & .988 .9 & .9900 \\
$\mathbf{4 . 0}$ & .9909 & .9918 & .9926 & .9933 & .9939 & .9948 & .9950 & .9955 & .9959 & .9963 \\
$\mathbf{5 . 0}$ & .9966 & .9970 & .9972 & .9975 & .9978 & .9980 & .9982 & .9984 & .9985 & .9986 \\
\hline
\end{tabular}

$$
p=2, \quad \lambda=2
$$

\begin{tabular}{ccccccccccc}
\hline \hline $\mathbf{x}$ & $\mathbf{. 0 0}$ &. $\mathbf{1 0}$ & $\mathbf{. 2 0}$ & $\mathbf{. 3 0}$ &. $\mathbf{4 0}$ & $\mathbf{. 5 0}$ & $\mathbf{. 6 0}$ & $\mathbf{. 7 0}$ & $\mathbf{. 8 0}$ & $\mathbf{. 9 0}$ \\
\hline $\mathbf{0}$ & .7400 & .7694 & .7959 & .8203 & .8426 & .8628 & .8809 & .8970 & .9114 & .9240 \\
$\mathbf{1 . 0}$ & .9351 & .9447 & .9531 & .9603 & .9665 & .9718 & .9763 & .9802 & .9834 & .9892 \\
$\mathbf{2 . 0}$ & .9885 & .9904 & .9920 & .9934 & .9945 & .9955 & .9962 & .9969 & .9974 & .9979 \\
$\mathbf{3 . 0}$ & .9983 & .9986 & .9988 & .9990 & .9992 & .9993 & .9994 & .9999 & .9996 & .999. \\
$\mathbf{4 . 0}$ & .9998 & .9998 & .9998 & .9999 & .9999 & .9999 & .9999 & .9999 & .9999 & 1.000 \\
$\mathbf{5 . 0}$ & 1.000 & 1.000 & 1.000 & 1.000 & 1.000 & 1.000 & 1.000 & 1.000 & 1.000 & 1.000 \\
\hline
\end{tabular}

$$
p=2, \quad \lambda=3
$$

\begin{tabular}{ccccccccccc}
\hline \hline $\mathbf{x}$ & $\mathbf{. 0 0}$ &. $\mathbf{1 0}$ & $\mathbf{. 2 0}$ & $\mathbf{. 3 0}$ & $\mathbf{. 4 0}$ & $\mathbf{. 5 0}$ & $\mathbf{. 6 0}$ & $\mathbf{. 7 0}$ & $\mathbf{. 8 0}$ & $\mathbf{. 9 0}$ \\
\hline $\mathbf{0 . 0}$ & .8400 & .8638 & .8818 & .8979 & .9121 & .9247 & .9357 & .9452 & .9535 & .9607 \\
$\mathbf{1 . 0}$ & .9668 & .9721 & .9765 & .9804 & .9836 & .9863 & .9886 & .9905 & .9921 & .9934 \\
$\mathbf{2 . 0}$ & .9946 & .9955 & .9963 & .9969 & .9975 & .9979 & .9983 & .9986 & .9988 & .9990 \\
$\mathbf{3 . 0}$ & .9992 & .9994 & .9995 & .9996 & .9996 & .9997 & .9998 & .9998 & .99998 & .9999 \\
$\mathbf{4 . 0}$ & .9999 & .9999 & .9999 & .9999 & 1.000 & 1.000 & 1.000 & 1.000 & 1.000 & 1.000 \\
$\mathbf{5 . 0}$ & 1.000 & 1.000 & 1.000 & 1.000 & 1.000 & 1.000 & 1.000 & 1.000 & 1.000 & 1.000 \\
\hline
\end{tabular}

The probability that an extended type III generalized logistic random variable $X$ lies in an interval $\left(\alpha_{1}, \alpha_{2}\right)$ is given as

$$
\operatorname{Pr}\left(\alpha_{1}<X<\alpha_{2}\right)=F_{X}\left(\alpha_{2}\right)-F_{X}\left(\alpha_{1}\right)
$$

for any real value of $p, \lambda$ and any given interval $\alpha_{1}<\alpha_{2}$. 


\section{The Moments of the Extended Type III Gen- eralized Logistic Distribution}

The moment generating function of the extended type III generalized logistic distribution is given as

$$
\begin{aligned}
M_{X}(t)=E[\exp \{t x\}] & =\int_{-\infty}^{\infty} \exp \{t x\} f_{X}(x) d x \\
& =\int_{-\infty}^{\infty} \exp \{t x\} \frac{\lambda^{p} \exp \{-p x\}}{B(p, p)(\lambda+\exp \{-x\})^{2 p}} d x \\
& =\frac{\lambda^{-t} \Gamma(p+t) \Gamma(p-t)}{\{\Gamma(p)\}^{2}}
\end{aligned}
$$

From the above equation (6), the mean and other moments of the extended type III generalized logistic distribution are obtained below. The cumulant generating function is obtained as

$$
\ln M_{X}(t)=-t \ln \lambda+\ln \Gamma(p+t)+\ln \Gamma(p-t)-2 \ln \Gamma(p)
$$

and the $r^{\text {th }}$ cumulant is given by

$$
\kappa_{r}(X)=\frac{d^{r}}{d t^{r}}[-t \ln \lambda]_{t=0}+\frac{d^{r}}{d t^{r}}[\ln \Gamma(p+t)+\ln \Gamma(p-t)]_{t=0} .
$$

The second term of the right hand side of equation (7) is called di-gamma function. The series expansion of this function as given in Copson (1962) is

$$
\psi^{r-1}(x)=(r-1) !\left\{(-1)^{r} \sum_{j=0}^{\infty}(j+x)^{-r}\right\}, \quad r \geqslant 2
$$

and

$$
\psi(x)=\sum_{j=0}^{\infty}(j+x)^{-1}, \quad r=1
$$

where

$$
\psi^{r-1}(z)=\frac{d^{r}}{d z^{r}}\{\ln \Gamma(z+1)\}
$$

Therefore

$$
\kappa_{r}(X)=(r-1) !(-1)^{r}\left\{\sum_{j=0}^{\infty}(j+p)^{-r}+(-1)^{r} \sum_{j=0}^{\infty}(j+p)^{-r}\right\}, \quad r \geqslant 2
$$


and

$$
\kappa_{r}(X)=-\ln \lambda-\sum_{j=0}^{\infty}(j+p)^{-1}+\sum_{j=0}^{\infty}(j+p)^{-1}=-\ln \lambda, \quad r=1 .
$$

Since the mean is obtained when $r=1$, the mean $\mu_{1}$ of the extended type III generalized logistic distribution is obtained as

$$
\mu_{1}=-\ln \lambda \text {. }
$$

The second cumulant of the random variable $X$ that has the extended type III generalized logistic distribution is

$$
\begin{aligned}
\kappa_{2}(X) & =\sum_{j=0}^{\infty}(j+p)^{-2}+\sum_{j=0}^{\infty}(j+p)^{-2} \\
& =2 \sum_{j=0}^{\infty}(j+p)^{-2} \\
& =2 \sum_{j=p}^{\infty} j^{-2} \\
& =2 \sum_{j=1}^{\infty} j^{-2}-\left.2 \sum_{j=1}^{p-1} j^{-2}\right|_{=0} \text { for symmetry }=\frac{\pi^{2}}{3} .
\end{aligned}
$$

The variance of $X$ is obtained as

$$
\sigma_{X}^{2}=\kappa_{2}(X)-\left\{\kappa_{1}(X)\right\}^{2}=\frac{\pi^{2}}{3}-(\ln \lambda)^{2} .
$$

The third cumulant of the random variable $X$ that has extended type III generalized logistic distribution is

$$
\kappa_{3}(X)=2(-1)^{3}\left\{\sum_{j=0}^{\infty}(j+p)^{-3}+(-1)^{3} \sum_{j=0}^{\infty}(j+p)^{-3}\right\}=0 .
$$

Let $\mu_{2}=\sigma_{X}^{2}$ and

$$
\mu_{3}=\kappa_{3}(X)-3 \kappa_{2}(X) \kappa_{1}(X)+2\left\{\kappa_{1}(X)\right\}^{3}=\pi^{2} \ln \lambda-2 \ln ^{3} \lambda .
$$

then the coefficient of skewness of the random variable $X$ that has extended type III generalized logistic distribution is

$$
\beta_{1}(X)=\frac{\mu_{3}^{2}}{\mu_{2}^{3}}=\frac{\left(\pi^{2} \ln \lambda-2 \ln ^{3} \lambda\right)^{2}}{\left(\frac{\pi^{2}}{3}-\ln ^{2} \lambda\right)^{3}} .
$$


The fourth cumulant of the random variable $X$ that has extended type III generalized logistic distribution is

$$
\begin{aligned}
\kappa_{4}(X) & =6\left\{\sum_{j=0}^{\infty}(j+p)^{-4}+(-1)^{4} \sum_{j=0}^{\infty}(j+p)^{-4}\right\} \\
& =12 \sum_{j=0}^{\infty}(j+p)^{-4}=12 \sum_{j=p}^{\infty} j^{-4} .
\end{aligned}
$$

Let $\mu_{4}=\kappa_{4}(X)-4 \kappa_{3}(X) \kappa_{1}(X)+6 \kappa_{2}\left\{\kappa_{1}(X)\right\}^{2}-3\left\{\kappa_{1}(X)\right\}^{4}$, then the coefficient of kurtosis of the random variable $X$ that has extended type III generalized logistic distribution is

$$
\beta_{2}(X)=\frac{\mu_{4}}{\mu_{2}^{2}} .
$$

\section{Some Theorems that Characterize the Ex- tended Type III Generalized Logistic Distri- bution}

We state some theorems and prove them in this section.

Definition 1. A continuously distributed random variable $X$ is said to have extended type I generalized logistic distribution if its probability density function is given as

$$
f_{X}(x ; \lambda, p)=\frac{p \lambda^{p} \exp \{-x\}}{(\lambda+\exp \{-x\})^{p+1}}, \quad-\infty<x<\infty, \quad p>0, \quad \lambda>0
$$

(see Olapade, 2004). When $p=1$, we have

$$
f_{X}(x ; \lambda)=\frac{\lambda \exp \{-x\}}{(\lambda+\exp \{-x\})^{2}}, \quad-\infty<x<\infty, \quad \lambda>0 .
$$

Equation (9) is what we refer to as extended logistic distribution density function which becomes ordinary logistic when $\lambda=1$.

Definition 2. A continuously distributed random variable $X$ is said to have extended type II generalized logistic distribution if its probability density function is given as

$$
f_{X}(x ; \lambda, p)=\frac{\lambda p \exp \{-p x\}}{(\lambda+\exp \{-x\})^{p+1}}, \quad-\infty<x<\infty, \quad p>0, \quad \lambda>0 .
$$


Theorem 1 Let $X_{1}, X_{2}, \ldots, X_{2 p-1}$ be extended logistic random variable sample of size $(2 p-1)$ having extended logistic probability density function

$$
f(x ; \lambda)=\frac{\lambda \exp \{-x\}}{(\lambda+\exp \{-x\})^{2}}, \quad-\infty<x<\infty, \quad \lambda>0 .
$$

and cumulative distribution function

$$
F(x)=\frac{\lambda}{\lambda+\exp \{-x\}}, \quad-\infty<x<\infty, \quad \lambda>0 .
$$

Then, the sample median $X_{p}$ has a symmetric generalized logistic distribution with parameters $\lambda$ and $p$.

Proof. From the theory of order statistics, the probability density function of the sample median $X_{p}$ in a sample of size $2 p-1$ is given by

$$
\begin{aligned}
f\left(x_{p}\right) & =\frac{(2 p-1) !}{(p-1) !(p-1) !}\{F(x)\}^{p-1}\{1-F(x)\}^{p-1} f(x) \\
& =\frac{\Gamma(2 p)}{\{\Gamma(p)\}^{2}}\left(\frac{\lambda}{\lambda+\exp \{-x\}}\right)^{p-1}\left(1-\frac{\lambda}{\lambda+\exp \{-x\}}\right)^{p-1} \frac{\lambda \exp \{-x\}}{(\lambda+\exp \{-x\})^{2}} \\
& =\frac{\lambda^{p}}{B(p, p)} \cdot \frac{\exp \{-p x\}}{(\lambda+\exp \{-x\})^{2 p}} .
\end{aligned}
$$

Since the equation (11) is the probability density function of a symmetric extended generalized logistic distribution, the proof is complete.

Theorem 2 Let $X$ be an extended logistic random variable, $X_{1}$ an extended type I generalized logistic random variable, $X_{2}$ an extended type II generalized logistic random variable and let $X_{3}$ be a random variable with probability density function f. Then

$$
X+X_{3} \stackrel{L}{=} X_{1}+X_{2}
$$

if $X_{3}$ has a symmetric extended generalized logistic distribution, where $\stackrel{L}{=}$ denotes equality in distribution and $X, X_{1}, X_{2}$ and $X_{3}$ are independent random variables.

Proof. Suppose $X$ is an extended logistic random variable with probability density function

$$
f_{X}(x, \lambda)=\frac{\lambda \exp \{-x\}}{(\lambda+\exp \{-x\})^{2}}, \quad-\infty<x<\infty, \quad \lambda>0
$$


then the characteristic function of $X$ is

$$
\phi_{X}(t)=\int_{-\infty}^{\infty} \exp \{i t x\} \frac{\lambda \exp \{-x\}}{(\lambda+\exp \{-x\})^{2}} d x=\lambda^{-i t} \Gamma(1+i t) \Gamma(1-i t) .
$$

Also suppose $X_{1}$ is an extended type I generalized logistic random variable with probability density function presented in the equation (8), then the characteristic function of $X_{1}$ is

$$
\phi_{X_{1}}(t)=\int_{-\infty}^{\infty} \exp \left\{i t x_{1}\right\} \frac{p \lambda^{p} \exp \left\{-x_{1}\right\}}{\left(\lambda+\exp \left\{-x_{1}\right\}\right)^{p+1}} d x_{1}=\lambda^{-i t} \frac{\Gamma(p+i t) \Gamma(1-i t)}{\Gamma(p)} .
$$

Similarly, since $X_{2}$ is an extended type II generalized logistic random variable with probability density function given in equation (10), then the characteristic function of $X_{2}$ is given as

$$
\phi_{X_{2}}(t)=\int_{-\infty}^{\infty} \exp \left\{i t x_{2}\right\} \frac{\lambda p \exp \left\{-p x_{2}\right\}}{\left(\lambda+\exp \left\{-x_{2}\right\}\right)^{p+1}} d x_{2}=\lambda^{-i t} \frac{\Gamma(1+i t) \Gamma(p-i t)}{\Gamma(p)} .
$$

Now,

$$
X+X_{3} \stackrel{L}{=} X_{1}+X_{2}
$$

implies that

$$
\phi_{X}(t) \phi_{X_{3}}(t)=\phi_{X_{1}}(t) \phi_{X_{2}}(t)
$$

that is

$$
\lambda^{-i t} \Gamma(1+i t) \Gamma(1-i t) \phi_{X_{3}}(t)=\frac{\lambda^{-2 i t} \Gamma(p+i t) \Gamma(1-i t) \Gamma(1+i t) \Gamma(p-i t)}{\{\Gamma(p)\}^{2}} .
$$

Therefore,

$$
\phi_{X_{3}}(t)=\frac{\lambda^{-i t} \Gamma(p+i t) \Gamma(p-i t)}{\{\Gamma(p)\}^{2}} .
$$

Since the equation (12) is the characteristic function of a symmetric extended generalized logistic distribution, the theorem is complete.

Theorem 3 The random variable $X$ is symmetric extended generalized logistic with probability density function $f$ given in the equation (5) if and only if $f$ satisfies the homogeneous differential equation

$$
(\lambda+\exp \{-x\}) f^{\prime}+p(\lambda-\exp \{-x\}) f=0,
$$

where prime denotes differentiation, $f$ denotes $f(x)$ and $f^{\prime}$ denotes $f^{\prime}(x)$. 
Proof. Suppose $X$ is a symmetric extended generalized logistic random variable, it is easily shown that the $f$ of the equation (5) satisfies the equation (13).

Conversely, let us assume that $f$ satisfies the equation (13). Separating the variables in the equation (13) we have

$$
\frac{f^{\prime}}{f}=\frac{p(\exp \{-x\}-\lambda)}{\lambda+\exp \{-x\}}=\frac{p \exp \{-x\}}{\lambda+\exp \{-x\}}-\frac{p \lambda}{\lambda+\exp \{-x\}} .
$$

Integrating, we have

$$
\ln f=-p x-2 p \ln (\lambda+\exp \{-x\})+\ln k,
$$

where $k$ is a constant. Obviously from the equation (14)

$$
f=\frac{k \exp \{-p x\}}{(\lambda+\exp \{-x\})^{2 p}} .
$$

The value of $k$ that makes $f$ a distribution function is $k=\lambda^{p} / B(p, p)$.

Theorem 3 is very important in the sense that if the equation (13) is transformed to make $x$ the subject, we have

$$
x=\ln \left\{\frac{p f-f^{\prime}}{\lambda\left(p f+f^{\prime}\right)}\right\} .
$$

Equation (15) can be regarded as "extended symmetric generalized logit transform" or an extended type III generalization of Berkson's logit transform in Berkson (1944).

\section{Estimation of the Parameters of the Extended Type III Generalized Logistic Distribution}

Before a probability distribution function can be fitted to any data, there is need to estimate the parameters in the model. Given a sample $X_{1}, X_{2}, \ldots, X_{n}$ of size $n$ from an extended type III generalized logistic distribution with probability density function

$$
f_{X}(x ; \mu, \sigma, \lambda, p)=\frac{\lambda^{p}}{\sigma B(p, p)} \cdot \frac{\exp \left\{-p\left(\frac{x_{i}-\mu}{\sigma}\right)\right\}}{\left[\lambda+\exp \left\{-\left(\frac{x_{i}-\mu}{\sigma}\right)\right\}\right]^{2 p}},
$$

where $\mu$ is the location parameter, $\sigma$ is the scale parameter, $p$ is the shape parameter and $\lambda$ is a shift or extension parameter. The likelihood function of 
the extended type III generalized logistic distribution is given as

$$
L(X ; \mu, \sigma, \lambda, p)=\frac{\lambda^{n p}}{\sigma^{n} B^{n}(p, p)} \cdot \frac{\exp \left\{-p \sum_{i=1}^{n}\left(\frac{x_{i}-\mu}{\sigma}\right)\right\}}{\prod_{i=1}^{n}\left[\lambda+\exp \left\{-\left(\frac{x_{i}-\mu}{\sigma}\right)\right\}\right]^{2 p}} .
$$

Taking the natural logarithm of both sides of $L(X ; \mu, \sigma, \lambda, p)$, we have

$$
\begin{aligned}
\ln L(X ; \mu, \sigma, \lambda, p)= & -n \ln B(p, p)+n p \ln \lambda-n \ln \sigma \\
& -p \sum_{i=1}^{n}\left(\frac{x_{i}-\mu}{\sigma}\right)-2 p \sum_{i=1}^{n} \ln \left[\lambda+\exp \left\{-\left(\frac{x_{i}-\mu}{\sigma}\right)\right\}\right] .
\end{aligned}
$$

To obtain the estimates of the parameters that maximize the likelihood function, we differentiate the logarithm of the likelihood function partially with respect to each of the parameters and set the derivatives to zero and solve for the parameters; here keep the parameter $p$ as a positive integer. Hence

$$
\begin{aligned}
\frac{\partial \ln L(X ; \mu, \sigma, \lambda, p)}{\partial \mu}= & \frac{n p}{\sigma}-\frac{2 p}{\sigma} \sum_{i=1}^{n} \frac{\exp \left\{-\left(\frac{x_{i}-\mu}{\sigma}\right)\right\}}{\left[\lambda+\exp \left\{-\left(\frac{x_{i}-\mu}{\sigma}\right)\right\}\right]} \\
\frac{\partial \ln L(X ; \mu, \sigma, \lambda, p)}{\partial \sigma}= & -\frac{n}{\sigma}+\frac{p \sum_{i=1}^{n}\left(x_{i}-\mu\right)}{\sigma^{2}} \\
& -\frac{2 p}{\sigma^{2}} \sum_{i=1}^{n} \frac{\left(x_{i}-\mu\right) \exp \left\{-\left(\frac{x_{i}-\mu}{\sigma}\right)\right\}}{\left[\lambda+\exp \left\{-\left(\frac{x_{i}-\mu}{\sigma}\right)\right\}\right]} . \\
\frac{\partial \ln L(X ; \mu, \sigma, \lambda, p)}{\partial \lambda}= & \frac{n p}{\lambda}-2 p \sum_{i=1}^{n} \frac{1}{\left[\lambda+\exp \left\{-\left(\frac{x_{i}-\mu}{\sigma}\right)\right\}\right]} .
\end{aligned}
$$

Since the equations $(16,17,18$ and 19) above are nonlinear in the parameters, we use numerical iterative method with the aid of computer programme to estimate the values of the parameters from a given sample.

\section{Application of the Extended Type III Gener- alized Logistic Model}

An agricultural scientist is interested in the distribution of the weight of the fruits from a new breed of orange tree. He harvested all the fruits of a new breed orange tree and weighed them, the weights are recorded and tabulated into a frequency distribution table as shown in Table 2. 
Table 2. Analysis of the weights of new breed of orange fruits

\begin{tabular}{ccccccc}
\hline \hline $\begin{array}{c}\text { Class } \\
\text { interval } \\
\text { of } \\
\text { weights }\end{array}$ & $\begin{array}{c}\text { Class } \\
\text { mid- } \\
\text { point } \\
\mathbf{x}\end{array}$ & $\begin{array}{c}\text { Class } \\
\text { frequency } \\
\text { observed }\end{array}$ & $\begin{array}{c}\text { Extended } \\
\text { type III } \\
\text { estimated } \\
\text { frequency }\end{array}$ & $\begin{array}{c}\text { Normal } \\
\text { estimated } \\
\text { frequency }\end{array}$ & $\begin{array}{c}\boldsymbol{\chi}^{\mathbf{2}} \text { extended } \\
\text { type III } \\
\text { generalized } \\
\text { logistic }\end{array}$ & $\begin{array}{c}\boldsymbol{\chi}^{\mathbf{2}} \\
\text { normal }\end{array}$ \\
\hline $0.1-0.5$ & 0.3 & 17 & 19.0 & 14.9 & 0.21 & 0.2960 \\
$0.6-1.0$ & 0.8 & 29 & 30.0 & 24.8 & 0.03 & 0.7113 \\
$1.1-1.5$ & 1.3 & 41 & 43.0 & 37.8 & 0.09 & 0.2709 \\
$1.6-2.0$ & 1.8 & 56 & 54.0 & 49.3 & 0.07 & 0.9105 \\
$2.1-2.5$ & 2.3 & 62 & 60.0 & 57.1 & 0.07 & 0.4205 \\
$2.6-3.0$ & 2.8 & 60 & 58.3 & 56.5 & 0.05 & 0.2168 \\
$3.1-3.5$ & 3.3 & 50 & 50.0 & 52.3 & 0.000 & 0.1011 \\
$3.6-4.0$ & 3.8 & 37 & 37.6 & 40.3 & 0.01 & 0.2702 \\
$4.1-4.5$ & 4.3 & 25 & 25.6 & 29.1 & 0.01 & .5777 \\
$4.6-5.0$ & 4.8 & 17 & 16.0 & 17.5 & 0.06 & 0.0143 \\
$5.1-5.5$ & 5.3 & 10 & 9.3 & 9.8 & 0.05 & 0.0041 \\
$5.6-6.0$ & 5.8 & 6 & 5.1 & 4.6 & 0.16 & 0.4261 \\
Total & & 410 & & & 0.81 & 4.2195 \\
\hline
\end{tabular}

A probability density function that will fit the hypothetical data is needed. The histogram of the data shows that the data is symmetric, hence we decided to fit the extended type III generalized logistic model and the normal model to them. The distribution of the data and the analysis are shown in Table 2.

The estimates of the parameters of the extended type III generalized logistic distribution which we obtained with the aid of computer programme are $(\hat{\mu}=$ $3.69, \hat{\sigma}=1.36, \hat{\lambda}=2.50, \hat{p}=2.00$ ) while those of the normal distribution are $(\hat{\mu}=2.623, \hat{\sigma}=1.41)$. The outcomes of the analysis are shown in Table 2.

The adequacy of the model is tested using the method of chi-square test. As shown in Table 2 above, $\chi_{\text {calculated }}^{2}$ for the extended type III generalized logistic model is 0.81 and that of the normal distribution is 4.2195 . From the statistical table of chi-square, $\chi_{(8,0.05)}^{2}=15.507$ and $\chi_{(10,0.05)}^{2}=18.307$. We conclude that the extended type III generalized logistic model is adequate and better for this data since its $\chi_{\text {calculated }}^{2}$ is less than $\chi_{\text {tabulated. }}^{2}$ and is smaller than the value of $\chi_{\text {calculated }}^{2}$ for the normal distribution.

\section{Conclusion}

We have demonstrated a method by which we obtained a symmetric extended generalized logistic distribution. The cumulative distribution function which 
does not have a closed form has been tabulated for some values of parameters $p$ and $\lambda$. The moments and characterizations of the distribution have been discussed and the paper ended with an application to some agricultural data. Further generalization of this distribution which contains four parameters to a five-parameter extended type III generalized logistic distribution is currently under study.

\section{Acknowledgment}

The author would like to express his sincere thanks to Prof. M. O. Ojo, the author's doctoral dissertation adviser at Obafemi Awolowo University for his advice always. Many thanks to the management of the African Institute of Mathematical Sciences, Cape Town, South Africa for the opportunity given to him to visit the centre where the computational parts of this paper were carried out. Sincere thanks also are due to the referees for their helpful comments and suggestions.

\section{References}

Balakrishnan, N.; Leung M. Y. (1988). Order statistics from the Type I generalized Logistic Distribution, Communications in Statistics - Simulation and Computation. 17, 25-50.

Berkson, J. (1944). Application of the logistic function to bioassay, Journal of the American Statistical Association, 39, 357-365.

Berkson, J. (1951). Why I prefer logits to probits, Biometrics 7, 327-339.

Berkson, J. (1953). A statistically precise and relatively simple method of estimating the bio-assay and quantal response, based on the logistic function, Journal of the American Statistical Association 48, 565-599.

Copson, E.T. (1962). An introduction to the theory of functions of a complex variable, Oxford University Press, London.

George, E.O.; Ojo, M.O. (1980). On a generalization of the logistic distribution, Annals of Statistical Mathematics, 32, 161-169.

Ojo, M.O. (1989). Analysis of some prison data, Journal of applied statistics, 16, 377-383.

Ojo, M.O. (1997). Some Relationships between the generalized logistic and other distributions, Statistica, 4, 573-579.

Ojo, M.O. (2002). Approximations to the distribution of the sum of the generalized logistic random varibles, Kragujevac Journal of Mathematics, 24, 135-145.

Ojo, M.O.; Olapade A.K. (2004). On a six-parameter generalized logistic distribution, Kragujevac Journal of Mathematics, 26, 31-38.

J. Statist. Res. Iran 3 (2006): 177-190 
Olapade, A.K. (2002). Some relationships between the Type II generalized logistic and other distributions, Journal of Statistical Research. 36, 213-218.

Olapade, A.K. (2004). On Extended Type I generalized logistic distribution, International Journal of Mathematics and Mathematical Sciences. 57, 3069-3074.

Olapade, A.K. (2005). On Negatively Skewed Extended Generalized Logistic Distribution, Kragujevac J. Math. Serbia. 27, 175-182.

Olapade, A.K.; Ojo, M.O. (2002). Characterizations of the Logistic Distribution, Nigerian Journal of Mathematics and Applications. 15, 30-36.

Pearson, K. (1934). Tables of the Incomplete Beta function, Cambridge University Press.

Verhulst, P.F. (1845). Recherches mathematiques sur la loi d'accresioement de la population, Academic Royale S'cience et Metres Bruxelee, 18, 1-38.

Wu J.W.; Hung W.L.; Lee H.M. (2000). Some Moments and Limit Behaviors of the Generalized Logistic Distribution with Applications, Proc. Natl. Sci. Counc. 24, 7-14.

\footnotetext{
Akintayo K. Olapade

Department of Mathematics, Obafemi Awolowo University.

Ile-Ife, Nigeria.

e-mail: akolapad@oauife.edu.ng
} 